\title{
Audiovestibular Loss of a Vascular Cause: A Distinction Should Be Made between Cochlear and Vestibular Symptoms
}

\author{
Antonio Pirodda, Maria Chiara Raimondi, Cristina Brandolini \\ Department of Experimental, Diagnostic and Specialty Medicine, University of Bologna, Bologna, Italy
}

\section{Dear Sir:}

An interesting paper recently published in Journal of Stroke ${ }^{1}$ thoroughly reviews the vascular causes of audiovestibular loss in order to appropriately deal with them. In particular, it is outlined how an isolated vertigo and/or an isolated sudden hearing loss depending on a selective infarction is unlikely although possible, and how the labyrinth is particularly prone to ischemia due its characteristics of terminal vasculature and high energy requirement.

Although all statements can be shared, in our opinion a distinction should be made between cochlear and vestibular symptoms.

Studies carried on by our group in the last two decades point out the role of functional causes related to hemodynamics in "idiopathic" sudden hearing loss (SHL), thus permitting to hypothesize the possible absence of arterial occlusive mechanisms in such cases: our observations, recently supported by $\mathrm{MRI}_{1}{ }^{2}$ propose an abrupt fall in blood pressure levels followed by a peripheral arterial spasm as a possible cause of SHL occurring in young people free from vascular risk factors; this may be explained considering a vascular dysregulation of primary origin., The above cited anatomic and functional characteristics of the labyrinth reliably support such a possibility. In the same direction, our studies on patients both submitted to different antihypertensive therapies ${ }^{5}$ and suffering from heart failure ${ }^{6}$ demonstrated an increased incidence of tinnitus referable to hemodynamic imbalance. These findings led us to propose cochlear symptoms as a warning mechanism for detecting cardiovascular impairments involving hemodynamics, ${ }^{7}$ as well as to hypothesize an original pathogenic mechanism for Meniere Disease. ${ }^{8}$
On the contrary, vestibular system is more diffusely represented in central nervous system, due to its connections with reticular substance, cerebellum, neuromuscular fuses etc.: accordingly, the concept of vascular vertigo is widespread in the literature and demonstrates a larger dependence on macro-vascular conditions as compared to auditory system. This difference can be observed in common ENT clinical practice; we recently discussed this aspect in commenting our 26-year lasting observation of a subject who had a SHL at 37, and subsequently underwent an audiological and cardiovascular follow-up always demonstrating the absence of appreciable vestibular and vascular impairments. ${ }^{9}$

In general terms, however, it can be concluded that the vestibular symptoms are more easily linked to a blood support derived from the middle arteries than to microcirculation, that in turn more strictly depends to local mechanisms and is less linked to atherosclerosis: even assuming all the reliability of the analysis made in the cited article, this difference must not be neglected when dealing with an isolated cochlear or vestibular disorder.

\section{References}

1. Kim HA, Lee $H$. Recent advances in understanding audiovestibular loss of a vascular cause. J Stroke 2017;19:61-66.

2. Sato $H_{1}$ Kawagishi $K$. Labyrinthine artery detection in patients with idiopathic sudden sensorineural hearing loss by 7-T MRI. Otolaryngol Head Neck Surg 2014;150:455-459.

3. Flammer J, Konieczka K, Flammer AJ. The primary vascular dysregulation syndrome implications for eye diseases. EPMA J 2013:4:14. 
4. Pirodda A, Brandolini C, Cassandro E, Borghi C. Primary vascular dysregulation syndrome: possible implications for inner ear acute diseases? Med Hypotheses 2015;85:586-587.

5. Borghi C, Brandolini C, Prandin MG, Dormi A, Modugno GC, Pirodda A. Prevalence of tinnitus in patients with hypertension and the impact of different antihypertensive drugs on the incidence of tinnitus: a prospective, single-blind, observational study. Curr Ther Res Clin Exp 2005;66:420-432.

6. Borghi C, Cosentino ER, Rinaldi ER, Brandolini C, Raimondi $M C$, Veronesi $M$, et al. Tinnitus in elderly patients and prognosis of mild-to-moderate congestive heart failure: a cross sectional study with a long-term extension of the clinical follow-up. BMCMed 2011;9:80.

7. Pirodda A, Cicero AF, Brandolini C, Borghi C. Inner ear symptoms: can we use them to approach cardiovascular diseases? Intern Emerg Med 2014;9:825-827.

8. Pirodda A, Brandolini C, Raimondi MC, Ferri GG, Modugno GC, Borghi C. Meniere's disease: update of etiopathogenetic the- ories and proposal of a possible model of explanation. Acta Clin Belg 2010;65:170-175.

9. Pirodda A, Brandolini C, Raimondi MC, Rinaldi ER, Borghi C. Inner ear acute damages and cardiocirculatory system: a case of sudden sensorineural hearing loss followed up by a 26year cardiovascular and audiologic monitoring. Acta Medica Mediterr 2016;32:863-864.

Correspondence: Cristina Brandolini Department of Experimental, Diagnostic and Specialty Medicine, University of Bologna, via Massarenti 9, Bologna, Italy

Tel: +390512143266

Fax: +39 0512143525

E-mail: cristina.brandolini@aosp.bo.it

Received: January 27, 2017

Revised: January 27, 2017

Accepted: May 7, 2017

The authors have no financial conflicts of interest. 